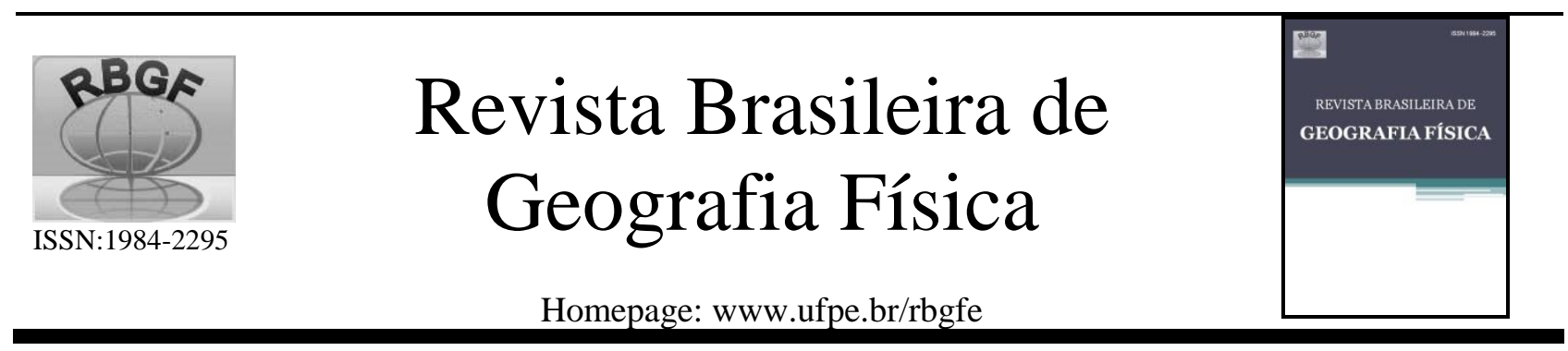

\title{
Sistema agroflorestal cafeeiro-abacateiro e seus efeitos na qualidade do solo
}

\author{
Bruno Salomão Pavan ${ }^{1}$; Rogério Melloni²*; Maria Inês Nogueira Alvarenga ${ }^{3}$; Gustavo Magno dos Reis \\ Ferreira $^{4}$
}

${ }^{1}$ Eng. Ambiental; Instituto de Recursos Naturais Universidade Federal de Itajubá e-mail: bruno.spavan@ @otmail.com ${ }^{2}$ Eng. Agrônomo; Instituto de Recursos Naturais; Universidade Federal de Itajubá; e-mail*: rogerio.melloni@gmail.com 3 Eng. Agrônoma; Instituto de Recursos Naturais; Universidade Federal de Itajubá; e-mail*: minesalvarenga@gmail.com ${ }^{4}$ Biólogo; Departamento de Biologia; Universidade Federal de Lavras; e-mail: gustavo_mrf@yahoo.com.br

Artigo recebido em 21/05/2018 e aceite em 28/08/2018

\section{R E S U M O}

Os sistemas agroflorestais (SAFs) são uma alternativa aos métodos tradicionais de cultivo, com maior aproveitamento dos serviços ecossistêmicos proporcionados pela interação entre as diferentes culturas. Entretanto, estudos dessa interação devem ser incentivados, de forma a selecionar a melhor combinação de culturas e manejo a ser aplicado. $\mathrm{O}$ objetivo desse trabalho foi avaliar o impacto de diferentes níveis de sombreamento do cafeeiro por abacateiros, nas qualidades física, química e microbiana do solo. Para isso, uma área de cultivo cafeeiro-abacateiro, localizada ao sul de Minas Gerais, foi dividida em três sub-áreas, conforme o nível de sombreamento proporcionado pelo abacateiro, sendo $0 \%$ sombreado (SP - sol pleno), 30\% sombreado (sombreamento mediano) e $89 \%$ sombreado (sombreamento intenso), com coleta de amostras do solo em duas profundidades, 0-10 cm e 10-20 cm. Os resultados mostraram que o SAF cafeeiro-abacateiro não afetou a qualidade física e química do solo. Contrariamente, a qualidade microbiana foi melhorada com o sombreamento, indicando maior benefício agroecológico com a implantação desse SAF.

Palavras-chave: agroecossistema, SAF, Coffee arabica L., Persea americana Mill.

\section{Agroforestry system coffee-avocado and its effect on soil quality}

\begin{abstract}
A B S T R A C T
Agroforestry systems (AFSs) are an alternative to traditional methods of cultivation, with greater use of the ecosystem services provided by the interaction between different cultures. However, studies of this interaction should be encouraged in order to select the best combination of crops and management applied. The objective of this work was to evaluate the impact of different levels of coffee shading by avocado trees on the physical, chemical and microbial qualities of the soil. For this, an area of coffee-avocado cultivation, located at south of Minas Gerais, was divided into three sub-areas, according to the level of shade provided by the avocado tree, being 0\% shaded (SP - full sun), 30\% shaded (mean shading) and $89 \%$ shade (intense shading), with soil samples collected at two depths, 0-10 cm and 10-20 $\mathrm{cm})$. The results showed that the coffee-avocado did not affect the physical and chemical quality of the soil. In contrast, the microbial quality was improved with shading, indicating a greater agroecological benefit with the implementation of this AFS.
\end{abstract}

Keywords: agroecosystem, AFS, Coffee arabica L., Persea americana Mill. 


\section{Introdução}

Sistemas agroflorestais (SAFs) apresentam uma contribuição à conservação da biodiversidade nativa por meio de uma conexão entre áreas preservadas e de cultivo, com características favoráveis para o solo e água, além de oferecer sustento às famílias que o administram (Vallejo et al., 2014; Oliveira et al., 2016; Sharma et al., 2016). Também promovem a estabilidade do microclima, mantendo a umidade do ar e do solo e reduzindo a dinâmica de liberação de $\mathrm{CO}_{2}$ (Carvalho et al., 2016).

A sustentabilidade da produção de café em SAFs requer compreensão das práticas de manejo, avaliando-se as interações dentro do contexto agroecológico local e seus possíveis impactos nos serviços ecossistêmicos (Boreux et al., 2016). Os atributos relacionados à qualidade do solo apresentam-se melhores em SAFs, quando comparados a sistemas de manejo do cafeeiro em sol pleno (Oliveira et al., 2016; Melloni et al., 2018). Além disso, Cerda et al. (2017) demonstram que os SAFs apresentam maiores serviços ao ecossistema, como controle de doenças e pragas, aumento da produtividade, melhoria da fertilidade do solo e maior sequestro de carbono, além de proporcionar maiores taxas de polinização do cafezal (Nesper et al., 2017; Boreux et al., 2016). Quanto à estrutura do solo, o cultivo do cafeeiro em consórcio com espécies arbóreas demonstra uma maior porosidade e saturação da condutividade hidráulica pelo solo quando comparado ao cultivo convencional, o que garante maior resistência a processos erosivos e produção mais sustentável (Gaitán et al., 2016). Oliveira et al. (2016) avaliaram os efeitos promovidos por diferentes intensidades de sombreamento do café por araucária (Araucaria angustifolia $\mathrm{L}$,) na macrofauna, qualidade física do solo e, consequentemente, produção de grãos, no sul de Minas Gerais, Brasil. Os autores verificaram que o cultivo de cafeeiro em gleba medianamente sombreada possibilitou, além de produção de grãos igual a do cafeeiro cultivado a pleno sol, melhorias na qualidade física, aumento no teor de matéria orgânica, na diversidade e riqueza da macrofauna do solo.

Como todo SAF, cultivos mistos de cafeeiro com outras culturas apresentam um complemento na fonte de renda para os agricultores, bem como para a estabilidade ambiental (Luedeling et al., 2014). Como exemplo, Alves et al. (2015) observaram viabilidade econômica do cultivo de cafeeiro com bananeira, principalmente pela última ser uma cultura de baixo custo.

Outra cultura que pode ser indicada para o sistema em conjunto com o cafeeiro é o abacateiro, principalmente devido a sua adaptação a diferentes climas e tipos de solo. Quando cultivado em consórcio com café, o abacateiro proporciona sombreamento eficiente, auxilia no estabelecimento das plântulas, mantém a qualidade do café, produz frutos com alto valor econômico e com baixo custo de produção, protege o solo da ação de chuvas, aumenta a biodiversidade, principalmente de aves, aumenta a disponibilidade de nutrientes e reduzem também a temperatura do solo (Govindappa e Elavarasan, 2014). No entanto, estudos relacionados à qualidade do solo devem envolver aspectos relacionados à física, química e microbiologia do solo (Paz-Ferreiro e Fu, 2013).

O cultivo do abacateiro em consórcio com o cafeeiro promove maior crescimento e rendimento do primeiro devido ao melhor manejo e maior sucesso na polinização cruzada entre as populações (Biazin et al., 2015). Entretanto, conforme esses autores, com o aumento da altura do abacateiro deve-se alterar o sistema de colheita dos frutos, já que pode interferir no processo de perda de frutos e ferimentos.

Apesar de evidentes os benefícios dos SAFs, estudos devem ser conduzidos visando otimização de processos e avaliação de seus efeitos na qualidade ambiental (Mbow et al., 2014). Sendo assim, pela ausência de estudos do impacto do abacateiro na qualidade do solo sob cultivo do cafeeiro, o presente trabalho teve como objetivo avaliar esse SAF sob diferentes níveis de sombreamento, em seus atributos físicos, químicos e microbiológicos.

\section{Material e métodos}

O trabalho foi conduzido no município de São Pedro da União, sul do estado de Minas Gerais, Brasil (Figura 1), localizado nas coordenadas 21009'29,33”S 46041'22,50”W. O clima na região é o tropical, com estações bem definidas, altitude variável de $1087 \mathrm{~m}$ a $1122 \mathrm{~m}$. A região está inserida no bioma Mata Atlântica com vegetação primária definida como Floresta Estacional Semidecidual, com solo dominante Argissolo Vermelho Amarelo distrófico (Brasil, 1983). 


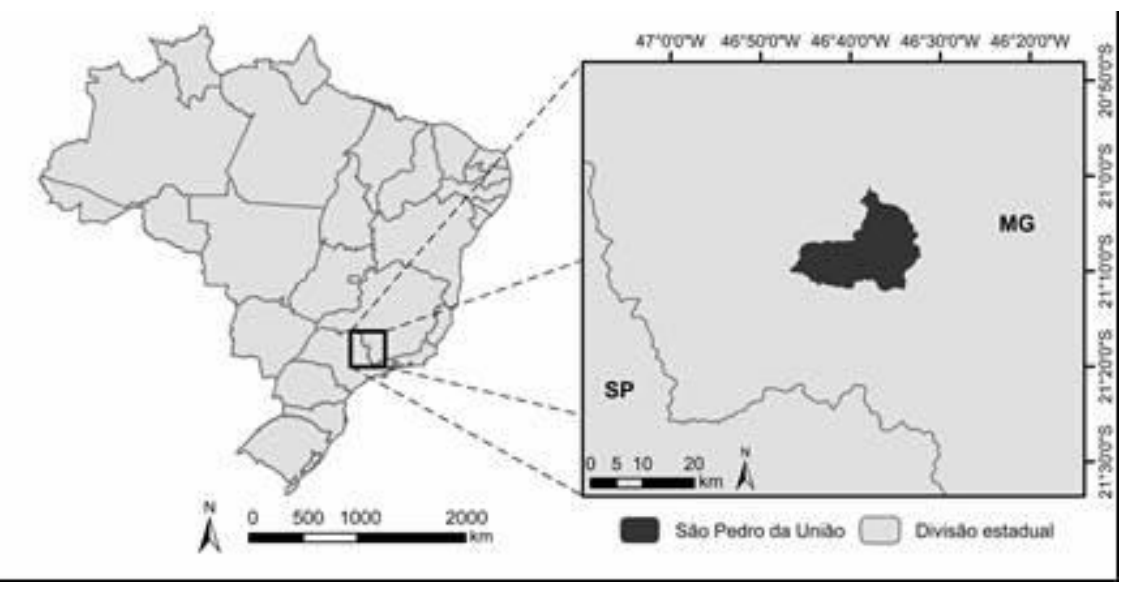

Figura 1. Localização geográfica da área de estudo, São Pedro da União no sul do estado de Minas Gerais.

O SAF estudado foi composto pelo cafeeiro (Coffea arabica L.) cultivar Catuaí Vermelho, com 20 anos de idade, em consórcio com abacateiro (Persea americana Mill.) cultivar Fortuna, com 15 anos de idade. Nesse SAF são utilizados fertilizantes fosfatados e nitrogenados, além da aplicação de adubos foliares e agrotóxicos como tiametozam e ciproconazol para controle de cigarras, e azoxistrobina e ciproconazol para controle de ferrugem do café. Para calagem é utilizado calcário dolomítico de três em três anos para elevar a saturação por bases a $70 \%$.

A área total de estudo foi composta por 2,7 ha e dividida em 3 subáreas, de acordo com o nível de sombreamento: a) 0,88 ha com cultivo do cafeeiro em sol pleno (SP), sem abacateiro; b) 0,66 ha com cultivo de cafeeiro em linhas alternadas com abacateiro, caracterizando o sombreamento mediano (SM) e c) 0,40 ha com cultivo de cafeeiro sob sombreamento intenso (SI). Os cafeeiros estão plantados com espaçamento de $1,0 \mathrm{~m}$ entre pés e 2,5 m entre as linhas. Já os abacateiros estão plantados em linha, com espaçamento de $10 \mathrm{~m}$ entre pés, variando o espaçamento entre as linhas, conforme os níveis de sombreamento. Para o SI a distância entre linhas é de 7,5 m e para o SM a distância entre linhas é de $15 \mathrm{~m}$, intercalando com os cafeeiros.

Para avaliar a variação de sombreamento em função da disposição dos abacateiros, foram feitas fotografias por meio de câmera digital instalada à altura de $0,15 \mathrm{~m}$ do chão, entre as linhas, fora da copa do cafeeiro (Figura 2). As diferentes imagens foram analisadas por meio do software Gap light analyzer-GLA 2.0 (Frazer et al., 1999).



Figura 2. Imagens do dossel: a) Sol Pleno (SP); b) Sombreamento mediano (SM) e c) Sombreamento intenso (SI). 
Para cada subárea foram coletadas três amostras compostas de solo, em duas profundidades: $0-10 \mathrm{~cm}$ e $10-30 \mathrm{~cm}$, por meio de enxada desinfestada com álcool $70 \%$ a cada amostra. Cada amostra composta foi formada por 5 amostras simples retiradas de forma intercalada e, posteriormente, homogeneizadas. Especificamente, o trabalho foi avaliado como esquema fatorial $3 \times 2$, em blocos casualizados, sendo os fatores: a) 3 níveis de sombreamento e b) 2 profundidades do solo, com 3 repetições por tratamento.

As amostras de solo foram acondicionadas em sacos plásticos, armazenadas a $4^{\circ} \mathrm{C}$, antes das análises físicas, químicas e microbiológicas. A estabilidade dos agregados e textura foram determinadas segundo Teixeira et al. (2017). Para as análises químicas foram avaliados: $\mathrm{pH}$ em água na relação 1:2:5 (solo:água), $\mathrm{Ca}, \mathrm{Mg}$ e $\mathrm{Al}$ trocáveis extraídos com $\mathrm{KCl} 1 \mathrm{~N}$, analisados por titulometria, $\mathrm{P}$ e K extraídos pelo método Mehlich1 e analisados por colorimetria e fotometria de chama respectivamente, e o carbono orgânico foi determinado por colorimetria, utilizando o método de Walkley e Black (Teixeira et al., 2017).

Os atributos microbiológicos analisados foram a atividade microbiana do solo, pelo método proposto por Anderson (1992), carbono da biomassa microbiana (Ferreira et al., 1999) e quociente metabólico $\left(q \mathrm{CO}_{2}\right)$, calculado pela relação entre a respiração basal do solo e o carbono da biomassa microbiana (Anderson e Domsch, 1993).

Para avaliar o pressuposto de normalidade dos dados, foi realizado o teste de Shapiro-Wilk utilizando a aplicação Action do software Excel $^{\circledR}$. Em seguida, os dados foram submetidos à análise de variância (ANOVA) e teste de Scott-Knott (5\%) para avaliar a diferença das médias, utilizando o software estatístico Sisvar ${ }^{\circledR}$ (Ferreira, 2011). Para a análise de componentes principais (PCA) foi utilizado o software Statistica 7.0 (Statsoft, 2004).

\section{Resultados e discussão}

A porcentagem de cobertura do dossel apresentou os valores de $0 \%$ de cobertura no cultivo do cafeeiro sob sol pleno (SP), 30\% de cobertura em cultivo do cafeeiro sob sombreamento mediano (SM) e $89 \%$ de cobertura em cultivo sob sombreamento intenso (SI) (Figura 1a, b e c). Baliza et al. (2012), observaram desempenhos fotossintéticos variáveis sob os níveis 35,50 e $65 \%$ de sombreamento, com melhor crescimento até $65 \%$. Em SAF cafeeiro-araucária (Oliveira et al., 2016), onde observou-se sombreamento de até $94 \%$ em sombreamento intenso e até $84 \%$ em sombreamento mediano, a produção nesse último sistema não diferiu do cultivo do cafeeiro em sol pleno, evidenciando o benefício agroecológico desse sistema de manejo.

Verifica-se que o valor obtido no presente estudo para sombreamento intenso com abacateiro (89\%) foi muito semelhante àquele obtido por esses últimos autores, em SAF com cafeeiroaraucária.

Houve interação significativa $(\mathrm{p}<0,05)$ entre os fatores níveis de sombreamento e profundidade do solo somente para os atributos atividade microbiana, carbono da biomassa microbiana, quociente metabólico e matéria orgânica do solo (Tabela 1).

Tabela 1. Médias de atividade microbiana (ATV), quociente metabólico $\left(q \mathrm{CO}_{2}\right)$, carbono da biomassa microbiana (CBM) e matéria orgânica (MO), nos níveis de sombreamento sol pleno (SP), sombreamento médio (SM) e sombreamento intenso (SI), nas diferentes profundidades.

\begin{tabular}{|c|c|c|c|c|c|c|c|c|}
\hline \multirow[t]{2}{*}{$\begin{array}{c}\text { Sombrea- } \\
\text { mento }\end{array}$} & \multicolumn{2}{|c|}{$\begin{array}{c}\text { ATV } \\
\mathrm{mg} \mathrm{CO}_{2} / \mathrm{g} \text { solo } \\
\text { seco. } 10 \text { dias } \\
\end{array}$} & \multicolumn{2}{|c|}{$\begin{array}{c}q \mathrm{CO}_{2} \\
\mu \mathrm{g} \mathrm{CO}_{2} / \mu \mathrm{g} \mathrm{C} / \mathrm{g} \text { solo } \\
\text { seco.dia } \\
\end{array}$} & \multicolumn{2}{|c|}{$\begin{array}{c}\text { CBM } \\
\mathrm{mg} \mathrm{C} / \mathrm{g} \text { de C solo seco }\end{array}$} & \multicolumn{2}{|c|}{$\begin{array}{c}\mathrm{MO} \\
\mathrm{dag} / \mathrm{dm}^{3}\end{array}$} \\
\hline & $0-10 \mathrm{~cm}$ & $10-30 \mathrm{~cm}$ & $0-10 \mathrm{~cm}$ & $10-30 \mathrm{~cm}$ & $0-10 \mathrm{~cm}$ & $10-30 \mathrm{~cm}$ & $0-10 \mathrm{~cm}$ & $10-30 \mathrm{~cm}$ \\
\hline SP & $0,82 \mathrm{aA}$ & $0,02 \mathrm{bB}$ & $2,15 \mathrm{aA}$ & $0,03 \mathrm{bB}$ & $0,39 \mathrm{bB}$ & $0,67 \mathrm{aA}$ & $4,83 \mathrm{aA}$ & 2,67 aB \\
\hline SM & $0,64 \mathrm{bA}$ & $0,01 \mathrm{bB}$ & $1,07 \mathrm{bA}$ & $0,03 \mathrm{bB}$ & $0,60 \mathrm{aA}$ & $0,54 \mathrm{bA}$ & $4,35 \mathrm{bA}$ & 2,61 aB \\
\hline SI & $0,31 \mathrm{cA}$ & $0,07 \mathrm{aB}$ & $0,57 \mathrm{cA}$ & $0,51 \mathrm{aA}$ & $0,55 \mathrm{aA}$ & $0,13 \mathrm{cB}$ & $3,80 \mathrm{cA}$ & $2,57 \mathrm{aB}$ \\
\hline $\mathrm{CV}(\%)$ & \multicolumn{2}{|c|}{7,17} & \multicolumn{2}{|c|}{33,33} & \multicolumn{2}{|c|}{11,77} & \multicolumn{2}{|c|}{5,81} \\
\hline
\end{tabular}

Médias seguidas da mesma letra minúscula nas colunas e maiúsculas nas linhas não diferem entre si pelo teste de Scott-Knott a $5 \%$ de significância. CV $(\%)$ = Coeficiente de variação. 
Com o aumento do nível do sombreamento, verificou-se aumento do carbono da biomassa microbiana e redução da atividade microbiana, o que proporcionou redução do quociente metabólico $q \mathrm{CO}_{2}$, mesmo observando-se redução da matéria orgânica do solo do sistema SP para SI. Ao se comparar as diferentes profundidades do solo, pôde-se observar uma redução da atividade microbiana, $q \mathrm{CO}_{2}$ e matéria orgânica da camada de 0-10 para a $10-30 \mathrm{~cm}$, independentemente do nível do sombreamento. Já o carbono da biomassa microbiana na camada superficial do solo apresentou menores valores em cultivo de cafeeiro SP e maiores valores em cultivo de cafeeiro SI.

Conforme observado, maior teor de matéria orgânica foi obtida no SP, em relação aos SAFs (Tabela 1). No entanto, Oliveira et al. (2016), em SAF araucária-cafeeiro, demonstraram que a maior densidade das árvores nos SAFs interfere na passagem da luz solar, o que pode influenciar na degradação do material orgânico adicionado ao solo, reduzindo esse processo. Os processos de decomposição são influenciados pelas variações sazonais, principalmente da temperatura, sendo acelerados durante os meses mais quentes e desacelerados durante os meses mais frios. A queda de material orgânico proporcionado pelo SAF abacateiro-cafeeiro é contínua durante o ano, o que promoveria acúmulo de serapilheira e mudanças da qualidade na superfície do solo, como aumento da biomassa microbiana (Costa et al., 2017; Lima et al., 2015).

Camadas mais profundas do solo tendem a apresentar uma diminuição da biomassa microbiana e também uma diminuição da fertilidade, principalmente em relação ao $\mathrm{C}, \mathrm{N}$, $\mathrm{NO}_{3}{ }^{-}$e $\mathrm{NH}_{4}{ }^{+}$(Li et al., 2017). Entretanto, regiões mais profundas não devem ser negligenciadas nos estudos de qualidade do solo, pois mesmo com a redução da atividade biológica, essa região apresenta ainda uma importante função ecológica, relacionada aos ciclos dos nutrientes e ao sequestro de $\mathrm{C}$ do solo (Leeuwen et al., 2017).

Os valores de $q \mathrm{CO}_{2}$ estão relacionados à estabilidade ambiental, sendo os menores para situações de menor estresse microbiano (Liao \& Xie, 2006; Zhou et al., 2017), conforme se observou para os sistemas cafeeiro-abacateiro SM e SI (Tabela 1), mesmo apresentando menor teor de matéria orgânica em relação ao cafeeiro SP. Assim, pode-se supor que a qualidade do material depositado pelo abacateiro seja diferente daquele depositado somente pelo cafeeiro, o que pode conferir maiores serviços ecossistêmicos ao ambiente (Boreux et al., 2016).

Segundo Souza et al. (2016), sistemas de plantio de cafeeiro em sol pleno podem reduzir a qualidade do solo. Comprovando esse fato, no presente estudo, o cafeeiro nos sistemas SM e SI apresentou melhores valores de atributos microbiológicos (Tabela 1), os quais são importantes indicadores de condições ambientais (Pires et al., 2016; Yan et al., 2015; Geisseler e Scow, 2014).

Os atributos físicos e químicos do solo não apresentaram interação significativa $(p>0,05)$ entre os diferentes níveis de sombreamento e as profundidades do solo, estando os resultados das médias totais dessas análises apresentados na tabela 2.

Tabela 2. Médias totais de diâmetro médio geométrico (DMG), diâmetro médio ponderado (DMP), pH em água, potássio $(\mathrm{K})$; cálcio $(\mathrm{Ca})$; fósforo $(\mathrm{P})$; magnésio $(\mathrm{Mg})$; hidrogênio $(\mathrm{H})$; soma de bases $(\mathrm{SB})$, capacidade de troca catiônica (CTC), saturação por bases (V\%) nos diferentes níveis de sombreamento: sol pleno (SP), sombreamento médio (SM) e sombreamento intenso (SI) e profundidades 0-10 e 10-30 cm.

\begin{tabular}{|c|c|c|c|c|c|c|c|c|c|c|c|}
\hline & \multicolumn{11}{|c|}{ Atributos analisados } \\
\hline & DMG & DMP & $\mathrm{pH}$ & $\mathrm{K}$ & $\mathrm{Ca}$ & $\mathrm{P}$ & $\mathrm{Mg}$ & $\mathrm{H}$ & SB & CTC & $\mathrm{V}(\%)$ \\
\hline & \multicolumn{11}{|c|}{ Níveis de sombreamento } \\
\hline SP & $3,83 \mathrm{a}$ & $4,59 \mathrm{a}$ & $5,40 \mathrm{a}$ & $4,10 \mathrm{a}$ & $61,00 \mathrm{a}$ & $6,16 \mathrm{a}$ & $19,66 \mathrm{a}$ & $34,83 \mathrm{a}$ & $85,23 \mathrm{a}$ & $123,23 \mathrm{a}$ & $66,83 \mathrm{a}$ \\
\hline SM & $3,42 \mathrm{~b}$ & $4,35 \mathrm{~b}$ & $5,30 \mathrm{a}$ & $5,41 \mathrm{a}$ & $65,50 \mathrm{a}$ & $7,17 \mathrm{a}$ & $18,16 \mathrm{a}$ & $38,0 \mathrm{a}$ & $78,70 \mathrm{a}$ & $120,25 \mathrm{a}$ & $62,33 \mathrm{a}$ \\
\hline SI & $3,93 \mathrm{a}$ & $4,60 \mathrm{a}$ & $5,61 \mathrm{a}$ & $4,88 \mathrm{a}$ & $53,50 \mathrm{a}$ & $5,50 \mathrm{a}$ & $18,00 \mathrm{a}$ & $43,16 \mathrm{a}$ & $76,38 \mathrm{a}$ & $111,21 \mathrm{a}$ & $67,83 \mathrm{a}$ \\
\hline \multicolumn{12}{|c|}{ Profundidade do solo } \\
\hline $0-10 \mathrm{~cm}$ & $3,69 \mathrm{a}$ & $4,51 \mathrm{a}$ & $5,48 \mathrm{a}$ & $5,16 \mathrm{a}$ & $74,11 \mathrm{a}$ & $8,11 \mathrm{a}$ & $22,33 \mathrm{a}$ & $38,55 \mathrm{a}$ & $103,40 \mathrm{a}$ & $136,24 \mathrm{a}$ & $70,55 \mathrm{a}$ \\
\hline $10-30 \mathrm{~cm}$ & $3,77 \mathrm{a}$ & $4,52 \mathrm{a}$ & $5,38 \mathrm{a}$ & $4,44 \mathrm{a}$ & $45,77 \mathrm{~b}$ & $4,44 \mathrm{~b}$ & $14,88 \mathrm{~b}$ & $38,77 \mathrm{a}$ & $56,80 \mathrm{~b}$ & $100,22 \mathrm{~b}$ & $60,77 \mathrm{~b}$ \\
\hline $\mathrm{CV}(\%)$ & 9,61 & 4,01 & 5,78 & 17,09 & 19,03 & 38,71 & 31,49 & 17,31 & 19,19 & 16,32 & 14,2 \\
\hline
\end{tabular}

Médias seguidas da mesma letra minúscula nas colunas não diferem entre si pelo teste de Sckott-Knott a $5 \%$ de significância. $\mathrm{CV}(\%)=$ Coeficiente de variação. 
Os níveis de sombreamento afetaram somente os atributos físicos DMG e DMP, sem interferência nos atributos químicos da qualidade do solo (Tabela 2). No entanto, maiores valores de COT, $\mathrm{Ca}, \mathrm{P}, \mathrm{Mg}, \mathrm{SB}, \mathrm{CTC}$ e V\% foram encontrados na camada superior do solo, de 0-10 $\mathrm{cm}$, quando comparados aos da camada inferior. Tal resultado, possivelmente se deve ao fato da correção da fertilidade da camada de 0-10 cm, comumente realizada.

Para os atributos DMG e DMP, os maiores valores foram obtidos no cafeeiro SP e SI, comparado ao SM, portanto, sem relação com o teor de matéria orgânica do solo. Esse resultado contraria aqueles obtidos por Rossi et al. (2016), os quais concluíram que em sistemas conservacionistas com maior tempo de implantação e menor emprego de práticas culturais favorecem o acúmulo e a manutenção do carbono no solo, com consequentes aumentos dos valores de diâmetro médio ponderado e de diâmetro médio geométrico dos agregados.

Evidencia-se, portanto, a complexidade do processo de agregação nos sistemas estudados, sem relação com o teor de matéria orgânica ou carbono da biomassa microbiana, já que o cafeeiro SP apresentou maior e menor valores do primeiro e segundo, respectivamente, quando comparado aos demais sistemas (Tabela 1). Nesse sentido, apesar dos níveis de sombreamento não interferirem na qualidade química do solo, em função do manejo químico homogeneamente adotado nos três sistemas, há outros ganhos ambientais que devem ser destacados. Nesse sentifo, Boreux et al. (2016), ao estudarem SAFs de café com espécies arbóreas nativas, concluíram que o sombreamento, além de melhorar a produção do café, apresentou melhores serviços ecossistêmicos, como maior ciclagem de nutrientes, sequestro de carbono, controle de doenças, polinização e melhorias nas condições de microclima.

Os resultados da análise de componentes principais para a relação entre os diferentes níveis de sombreamento e atributos físicos, químicos e microbiológicos estão apresentados na figura 3, cujos autovalores acumulados nos dois principais componentes explicam $76,68 \%$ da variância total dos dados. Os componentes principais mostram a formação de 4 grupos distintos: I) os sistemas SP na profundidade $0-10 \mathrm{~cm}$ (SPP1) e SI na profundidade $0-10 \mathrm{~cm}$ (SIP1) com maiores relações com os principais atributos químicos e microbiológicos; II) sistema SM na profundidade 0-10 cm (SMP1) e relação com os atributos carbono da biomassa microbiana, fósforo e teor de argila; III) os sistemas SM na profundidade 10-20 $\mathrm{cm}$ (SMP2) e SI na profundidade 10-20 cm (SIP2) com relação inversa aos atributos químicos e microbiológicos, e IV) sistema SP na profundidade 10-20 cm (SPP2) com relação aos teores de areia e silte, DMG, DMP e $\mathrm{pH}$.

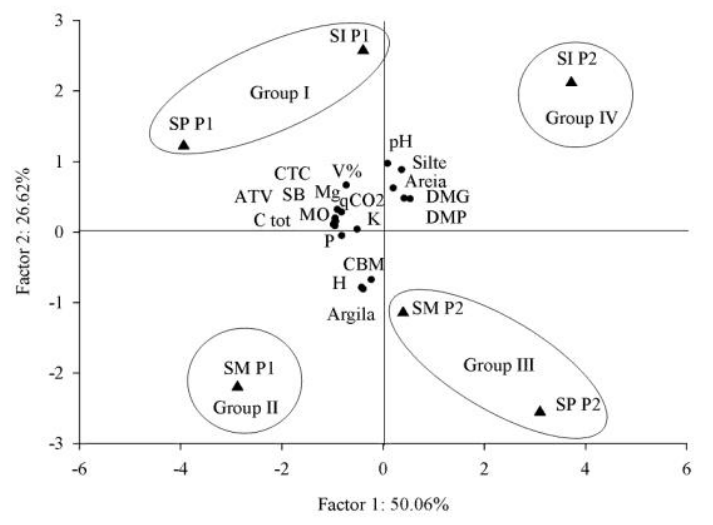

Figura 3. Resultado da análise de componentes principais para os atributos físicos, químicos e microbiológicos no sistema agroflorestal cafeeiro-abacateiro, em relação às duas profundidades (0-10 e 10$30 \mathrm{~cm}$ ) e três diferentes níveis de sombreamento, Cultivo em sol pleno na profundidade 0-10 cm (SP P1), cultivo em sol pleno na profundidade $10-30 \mathrm{~cm}$ (SL P2), Cultivo em sombreamento mediano na profundidade 0-10 cm (SM P1), cultivo em sombreamento mediano na profundidade 10-30 $\mathrm{cm}$ (SM P2), cultivo em sombreamento intenso na profundidade 0-10 cm (SI P1), cultivo em sombreamento intenso na profundidade 10-30 cm (SI P2), carbono da biomassa microbiana $(\mathrm{CBM})$, quociente metabólico $\left(q \mathrm{CO}_{2}\right)$, atividade microbiana (ATV), soma de bases (SB), matéria orgânica (MO), carbono orgânico total (C tot), saturação de bases (V\%), diâmetro médio geral dos agregados (DMG), diâmetro médio ponderado dos agregados (DMP), capacidade de troca catiônica (CTC). 
Dessa forma, conclui-se que houve homogeneidade da qualidade química na camada de 0-10 cm, independentemente do sistema, com redução dos teores de nutrientes na maior profundidade. Apesar do SP apresentar maior teor de matéria orgânica e maiores agregados, não se observou melhor qualidade microbiológica. Pelo contrário, nesse sistema houve redução da biomassa microbiana e maiores valores de quociente metabólico, indicando estresse microbiológico.

Nos sistemas com abacateiro, independente da densidade, houve aumento da biomassa microbiana, possivelmente em função das condições ambientais proporcionadas pelo sombreamento e qualidade do material orgânico adicionado ao longo do tempo. Dessa forma, pela manutenção da qualidade química e física, e maior qualidade microbiana (maior carbono da biomassa e menor $q \mathrm{CO}_{2}$ ) nesses ambientes, pode-se enfatizar maior benefício agroecológico, principalmente no SAF cafeeiro-abacateiro sob sombreamento intenso (SI).

Assim, estudos de qualidade ambiental envolvendo seus aspectos físicos, químicos e microbiológicos, permitem avaliação dos impactos de forma mais ampla, favorecendo, assim, o acompanhamento e a tomada de decisão para gestão e conservação da qualidade do solo em ambientes agrícolas (Velásquez et al., 2005).

\section{Conclusões}

O sistema agroflorestal cafeeiroabacateiro, independentemente de seus níveis de sombreamento, não afetou a qualidade física e química do solo.

O aumento do sombreamento proporcionado pelo abacateiro contribuiu para melhorias na qualidade microbiana do solo, com aumento da biomassa e redução do estresse microbiano $\left(q \mathrm{CO}_{2}\right)$.

\section{Agradecimentos}

Os autores agradecem a Coordenação de Aperfeiçoamento de Pessoal de Nível Superior (Capes) e a Fundação de Amparo à Pesquisa do Estado de Minas Gerais (Fapemig), pelo apoio.

\section{Referências}

Alves, E.P., Silva, M.L., Neto, S.N.O., Barella, T.P., Santos, R.H.S., 2015. Economic analisys of a coffee-banana system of a family-based agriculture at the atlantic forest zone, Brazil. Ciência e Agrotecnologia 39, 232-239.
Disponível: http://dx.doi.org/10.1590/S141370542015000300004. Acesso: 15 mar. 2018.

Anderson, T.H., Domsch, K.H., 1993. The metabolic quotient for $\mathrm{CO}_{2}\left(\mathrm{qCO}_{2}\right)$ as a specific activity parameter to assess the effects of environmental conditions, such as $\mathrm{pH}$, on the microbial biomass of forest soils. Soil Biology and Biochemistry 25, 393-395.Disponível: https://eurekamag.com/pdf/002/002524029.pdf. Acesso: 17 mar. 2018.

Baliza, D.P., Santos, M.O., Alves, J.D., Guimarães, R.J., Cunha, R.L., 2014. Metabolismo da sacarose em cafeeiros submetidos a diferentes níveis de sombreamento. Coffee Science 9, 445-455. Disponível:

http://dx.doi.org/10.25186/cs.v9i4.714. Acesso: 15 mar. 2018.

Biazin, B., Haileslassie, A., Zewdie, T., Mekasha, Y., Gebremedhin, B., Fekadu, A., Shewage, T., 2015. Smallholders avocado production systems and tree productivity in the souther highlands of Ethiopia. Agroforestry Systems 92, 127-137. Disponível: DOI 10.1007/s10457016-0020-2. Acesso: 21 mar. 2018.

Boreux, V., Vaast, P., Madappa, L.P., Cheppudira, K.G., Garcia, C., Ghazoul, J., 2016. Agroforestry coffee production increased by native Chade trees irrigation, and liming. Agronomy for sustainable development 36, 1-9. Disponível: http://DOI 10.1007/s13593-0160377-7. Acesso: 22 mar. 2018.

Brasil., 1983. Projeto RADAMBRASIL: levantamento dos recursos naturais. Folhas SF.23/24. Ministério das Minas e Energia, Rio de Janeiro/Vitória.

Carvalho, G.L., Maria, C.I., Sá, M.E., Alves, F.R.B., Schiavon, L.V., Sena, O.T., 2016. Agricultural and Forest Meteorology 224, 3039.

Disponível: http://dx.doi.org/10.1016/j.agrformet.2016.05.0 01. Acesso: 15 maio 2018.

Cerda, R., Allinne, C., Gary, C., Tixier, P., Harvey, C.A., Krolczyk, L., Mathiot, C., Clement, E., Aubertot J.N., Avelino, J., 2017. Effects of shade, altitude and management on multiple ecosystem services in coffee agroecosystems. European Journal of Agronomy 82, 308-319. Disponível:

http://dx.doi.org/10.1016/j.eja.2016.09.019. Acesso: 29 mar. 2018. 
Costa, P. M. O.; Araúo, M. A. G.; Souza-Motta, C. M.; Malosso, E., 2017. Dynamics of leaf litter and soil respiration in a complex multistrata agroforestry system, Pernambuno, Brazil. Environmental Development Science, 19, 11889-1203. Disponível: DOI 10.1007/s10668016-9789-4. Acesso: 31 jul. 2018.

Ferreira, A.S., Camargo, F.A.O., Vidor, C., 1999. Utilização de microondas na avaliação da biomassa microbiana do solo. Revista Brasileira de Ciência do Solo 23, 991-996.

Frazer, G.W., Canham, C.D., Lertzman, K.P., 1999. Gap light analyzer (GLA), version 2.0: imaging software to extract canopy structure and gap light transmission indices from truecolour fisheye photographs, users manual and program documentation. Disponível: <http://www.rem.sfu.ca/forestry/downloads/gap -light-analyzer.htm>. Acesso: 5 abr. 2018.

Gaitán, L., Armbrecht, I., Graefe, S., 2016. Throughfall and soil properties in shaded and unshaded coffee plantations and a secondary forest: a case study from Southern Colombia. Journal of agriculture and rural development in the tropics and subtropics 117, 309-321. Disponível: urn:nbn:de:hebis:342016101851061. Acesso: 12 abr. 2018.

Geisseler, D.; Scow, K. M., 2014. Long-term effects of mineral fertilizers on soil microorganisms - A review. Soil Biology and Biochemistry, 75, 54-63. Disponível: https://doi.org/10.1016/j.soilbio.2014.03.023. Acesso: 31 jul. 2018.

Govindappa, M., Elavarasan, K., 2014. Shade cum fruit yielding avocado under coffee ecosystem. International Letters of Natural Sciences 22, 6166. Disponível: //doi.org/10.18052/www.scipress.com/ILNS.27. 61. Acesso: 5 abr. 2018.

Leeuwen, J.P.V., Djukic, I., Bloem, J., Lehtinen, T., Hemerik, L., Ruiter, P.C., Lair, G.J., 2017. Effects of land use on soil microbial biomass, activity and community structure at different soil dephts in the Dabube floodplain. European Journal of Soil Biology 79, 14-20. Disponível: http://dx.doi.org/10.1016/j.ejsobi.2017.02.001. Acesso: 15 maio 2018.

Li, X., Sun, J., Wang, H., Li, X., Wang, J., Zhang, H., 2017. Changes in the soil microbial phospholipid fatty acid profile with depht in three soil types of paddy fields in China. Geoderma 290, 69-74. Disponível: http://dx.doi.org/10.1016/j.geoderma.2016.11.0 06. Acesso: 26 abr. 2018.

Lima, R. P.; Fernandes, M. M.; Fernandes, M. R. M.; Matricardi, E. A. T., 2015. Aporte e decomposição de serapilheira na Caatinga no Sul do Piauí. 22, 42-49. Disponível: http://dx.doi.org/10.1590/2179-8087.062013. Acesso: 31 jul. 2018.

Luedeling, E., Kindt, R., Huth, N., Koenig, K., 2014. Agroforestry systems in a changing climate - challenges in projecting future performance. Current Opinion in Environmental Sustainability 6, 1-7. Disponível: http://dx.doi.org/10.1016/j.cosust.2013.07.013. Acesso: 8 fev. 2018.

Mbow, C., Noordwijk, M.V., Luedeling, E., Neufeldt, H., Minang, P.A.; Kowero, G., 2014. Agroforestry solutions to address food security and climate change challenges in Africa. Current Opinion in Environmental Suistainability 6, 61-67. Disponível: http://dx.doi.org/10.1016/j.cosust.2013.10.014. Acesso: 8 fev. 2018.

Melloni, R.; Costa, N. R.; Melloni, E. G. P.; Lemes, M. C. S.; Alvarenga, M. I. N.; Neto, J. N., 2018. Sistemas agroflorestais cafeeiroaraucária e seu efeito na microbiota do solo e seus processos. Ciencia florestal, 28, 784-795. Disponível:

DOI: http://dx.doi.org/10.5902/1980509832392. Acesso: 31 jul. 2018.

Nesper, M.; Kueffer, C.; Krishnan, S.; Kushalappa, C. G.; Ghazoul, J., 2017. Shade tree diversity enhances coffee production and quality in agroforestry systems in the Western Ghats. Agriculture, Ecosystems and Environment, 247, 172-181.

Disponível: http://dx.doi.org/10.1016/j.agee.2017.06.024. Acesso: 31 jul. 2018.

Oliveira, C.C., Alvarenga, M.I.N., Melloni, R., Neto, J.N.P., Pinheiro, L.B.A., Melloni, E.G.P., Madeira, C.L., 2016. Revista Brasileira de Geografia Física 9, 1668-1676. Disponível: https://periodicos.ufpe.br/revistas/rbgfe/article/ view/233889. Acesso: 5 fev. 2018.

Pires, J.F., Ferreira, G.M.R., Reis, K.C., Schwan, R.F., Silva, C.F., 2016. Mixed yeasts inocula for simultaneous production of SCP and treatment of vinasse to reduce soil and fresh water pollution. Journal of Environmental Management 182, 455-463. Disponível: 
http://dx.doi.org/10.1016/j.jenvman.2016.08.00 6. Acesso: 15 maio 2018.

Rossi, C. Q.; Pereira, M. G.; Moura, O. V. T.; Almeida, A. P. C., 2016. Vias de formação, estabilidade e características químicas de agregados em solos sob sistemas de manejo agroecológico. Pesquisa Agropecuária Brasileira, 51, 1677-1685. Disponível: DOI: fe10.1590/S0100-204X2016000700068.

Acesso: 31 jul. 2018.

Sharma, N., Bohra, B., Pragya, N., Ciannella, R., Dobie, P., Lehmann, S., 2016. Bioenergy from agroforestry can lead to improved food security, climate change, soil quality, and rural development. Food and Energy Security 5, 165183. Disponível: http://10.1002/fes3.87. Acesso: 12 maio 2018.

Souza, G. S.; Dan, M. L.; Araújo, J. B. S., 2016. Qualidade física do solo sob cafeeiro conilon consorciado e em monocultivo. Coffee Science, 11, 180-186. Disponível: http://www.coffeescience.ufla.br/index.php/Cof feescience/article/download/1033/pdf_1033. Acesso: 31 jul. 2018.

Teixeira, P.C., Donagemma, G. K., Fontana, A., Teixeira, W. G. (Eds.), 2017. Manual de métodos de análise de solo, 3ed. Embrapa, Brasília. Disponível: http://ainfo.cnptia.embrapa.br/digital/bitstream/i tem/171907/1/Manual-de-Metodos-de-Analisede-Solo-2017.pdf. Acesso: 4 ago. 2018.
Valejjo, M., Casas, A., Blancas, J., Calles, A.I.M., Solis, L., Landa, S.R., Davila, P., Tellez, O., 2014. Agroforestry systems in the highlands of the Tehuacan Valley, México: indigenous cultures and biodiversity conservation. Agroforestry Systems 88, 125-140. Disponível: http://10.1007/s10457-013-9660-7. Acesso: 14 maio 2018.

Yan, N.; Marschner, P.; Cao, W.; Zuo, C.; Qin, W., 2015. Influence of salinity and water content on soil microorganisms. International soil and water conservation research, 3, 316-323. Disponível:

https://doi.org/10.1016/j.iswcr.2015.11.003. Acesso: 30 jul. 2018.

Zhou, H., Zhang, D., Wang, P., Liu, X., Cheng, K., Li, L., Zheng, J., Zhang, X., Zheng, J., Crowley, D., Zieten, L.V., 2017. Changes in microbial biomass and the metabilic quotient with biochar addition to agricultural soils: A meta-analysis. Agriculture, Ecosystems and Environment 239, 80-89. Disponível: http://dx.doi.org/10.1016/j.agee.2017.01.006. Acesso: 22 fev. 2018. 